\title{
Application of Dynamic Image Fusion Technology in Development of Innovation Capabilities of College Students Majoring in Art Design
}

\author{
https://doi.org/10.3991/ijet.v16i13.24043 \\ Zhe Wang \\ Jiaozuo University, Jiaozuo, China \\ wz15839128961@163.com
}

\begin{abstract}
Art design is based on art and images, in which dynamic images require pre-processing and fusion. This paper explores how to apply the dynamic image fusion technology in the development of the innovation capabilities of college students majoring in art design. The research results show that image sequences with different exposures can be directly formed into low dynamic range images through multi-exposure fusion, or the high dynamic range can be restored first, and then converted to a low dynamic range image through tone mapping. The main principles for innovation development in art design education include conformity, student-centeredness, and overall optimization. The dynamic image fusion enhancement algorithm is mainly divided into five functional modules, which are used to calculate multi-scale gradient, structure tensor, and target gradient for the fused image, solve the steepest descent method and output normalized images, respectively
\end{abstract}

Keywords - An art design major, tone mapping, image normalization, innovation capabilities, multi-exposure fusion

\section{Introduction}

There are many ways for humans to transmit information, but the most intuitive and important way is to use images $[1,2]$. Image information feels clear and easy to understand and contains large amount of information, so it is very important in all aspects of production and life [3]. Real scenes are usually expressed in the form of analog signals, but now, with the rapid development of computer technology, the analog signals in the original images obtained can be digitally analyzed and processed $[4,5]$. The image resolution supported by current shooting and display equipment is getting higher and higher, and high-resolution images are bound to be the mainstream in the image field in the future [6]. Some researchers have applied the heterogeneous parallel acceleration technology in the field of dynamic image processing to effectively improve the processing speed of the algorithm with regard to high-resolution images, and also combined it with the denoising technology and high dynamic range processing technology to accelerate the recording of the dynamic range imaging [7]. 
In recent years, the art design education and industry have seen an "explosive" development [8]. Many students have chosen art design as their major in colleges and universities. While having a lot in common with other college students, those majoring in art design also have distinctive personalities that others do not $[9,10]$. Developing their innovation capabilities in art design will help them create better design works; in addition, through such cultivation, colleges and universities can also find out more feasible and effective ways to educate these students [11, 12].

In terms of image fusion, some researchers have designed a new parallel optimization scheme for the algorithm in response to the problem of unbalanced load in the implementation of the face detection technology [13]. Optimization methods such as coarse-grained parallelism, dynamic mapping of threads and data, and global and local queues have significantly improved the performance of the algorithm implemented on the central processing unit $[14,15]$. In the study of the dynamic image denoising process, some researchers have found that low dynamic range images are featured with noise in lower exposure, and blur in higher exposure [16]. The method of using wavelet shrinkage to denoise the image sequence for multi-exposure fusion is to use a new weighting scheme to reduce the impacts on the fusion effect from the sensor noise and noise generated during the multi-exposure fusion [17, 18]. This paper explores how to apply the dynamic image fusion technology in the development of the innovation capabilities of college students majoring in art design.

\section{Research on the Image Fusion Acceleration Technologies}

\subsection{Parallel acceleration technology for image processors}

With the continuous development of the semiconductor technology, the research and application of image processors have also developed rapidly [19]. Besides having the traditional graphics rendering function, modern image processors are also providing support in various technological fields such as image processing, virtual reality, artificial intelligence and biomedicine [20]. With the continuous enhancement of the computing power of the image processors and the further popularization of the image processor programming technology, image processors will be needed in more and more fields to improve their work efficiency in the future [21, 22]. Like a central processing unit (CPU), an image processor communicates with the outside world through a bus, and it also has its own cache system and computing unit, but these two are applied in different scenarios and to process different objects [23]. The image processor is applied in simple scenarios and mainly for graphics rendering. It deals with data computing operations that are not dependent on each other and can be highly parallel, and that involve a huge amount of data [24, 25]. The floating-point computing capacity of the image processor is much stronger and its bandwidth is much broader than those of the CPU, so it is easier to achieve parallelism, and the broad bandwidth ensures the timeliness of its data transmission [26].

The kernel program of the image processor deals with functions that contain large amounts of data and that are independent of each other and have simple logical rela- 
tionships. However, as a device, the processor usually does not have the ability to input and output data. It needs to first transfer the data on the host to the device, and the device processes the data received by executing the kernel program [27, 28]. Figure 1 shows the scheme flow of the dynamic range imaging technology. As can be seen, image sequences with different exposures can be directly formed into low dynamic range images through multi-exposure fusion, or high dynamic range images can be restored first, and then low dynamic range images can be formed through tone mapping. Figure 2 is a schematic diagram of the image processor execution model. First, the platform and the device are determined, and the context and command queue are created, and then data are transferred to the device, which processes and sends back the data by executing the kernel program.

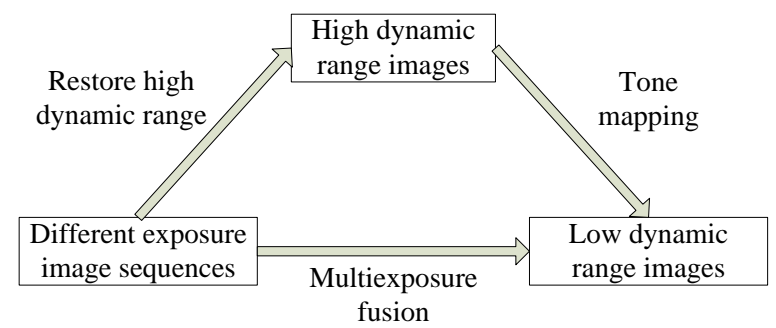

Fig. 1. Dynamic range imaging technology scheme flow

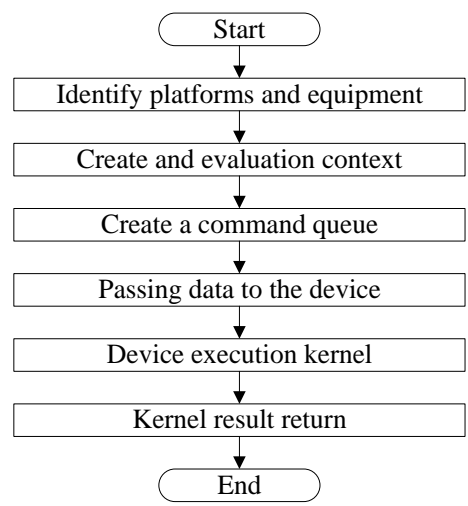

Fig. 2. Schematic diagram of image processor execution model

\subsection{Image multi-exposure fusion algorithm}

In order to achieve a better image fusion effect, it is necessary to denoise the low dynamic range image sequences acquired under different exposures before having them processed by the multi-exposure fusion algorithm. The commonly used denoising methods include bilateral filtering and non-local average filtering [29, 30]. Figure 3 is the implementation framework for multi-exposure fusion. Its core is to assign different weights to the image sequences with different exposures based on 
image features, and then fuse the brightness image sequences into an image with complete scene information and extended dynamic range based on the weights. The main steps include calculating the image features and the weights required for fusion, and weighting the images to obtain the fused image. In multi-exposure fusion, highquality pixels in the images should be selected and combined on a weighted basis through some methods [31].

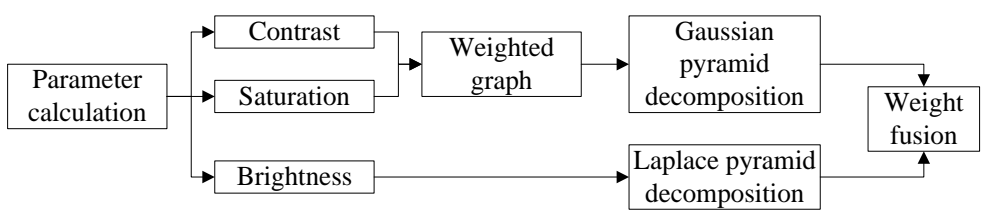

Fig. 3. Multi-exposure fusion implementation framework

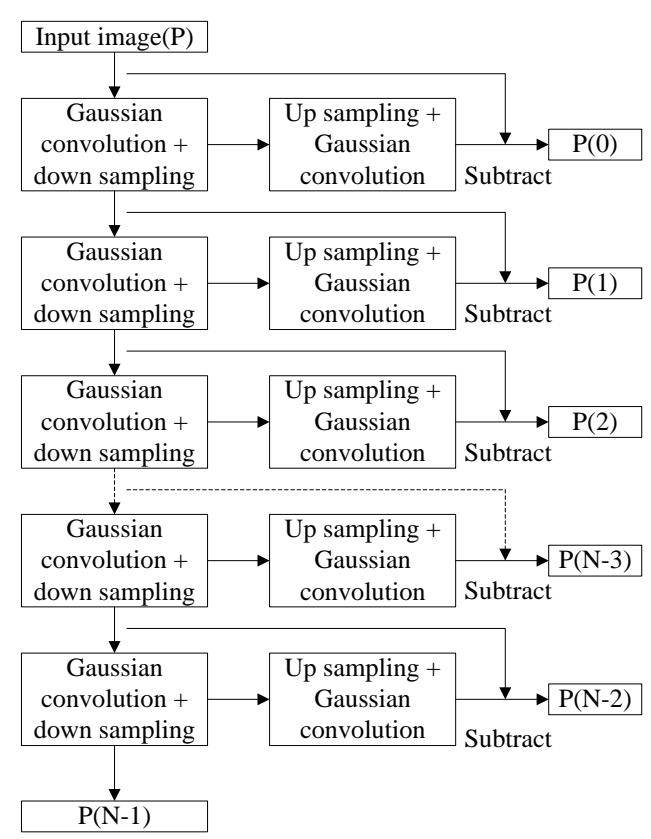

Fig. 4. Flowchart of the Laplacian pyramid construction

Figure 4 is a flow chart of how a Laplacian pyramid is constructed. With the original image as the input image, first perform Gaussian convolution and down-sampling of the input image to obtain the image data, and then perform up-sampling and Gaussian convolution of the image data to obtain an image. After the weighted result of each layer is obtained, the brightness result map needs to be reconstructed from the weighted result pyramid. Figure 5 is a flow chart of the brightness reconstruction based on the Laplacian pyramid. Starting from the N-th layer at the top of the pyramid, the image on the $\mathrm{N}$-th layer is used as the input image. After the input image is up-sampled and Gaussian convolved, it is combined with the image on the N-1-th 
layer into a new image on the N-1-th layer. After that, up-sampling and Gaussian convolution are performed again on the new image on the N-1-th layer, and the convolution result is combined with the image on the $\mathrm{N}$-2-th layer to form a new one on the N-2-th layer, and so on, until a new image is obtained at the bottommost layer of the pyramid.

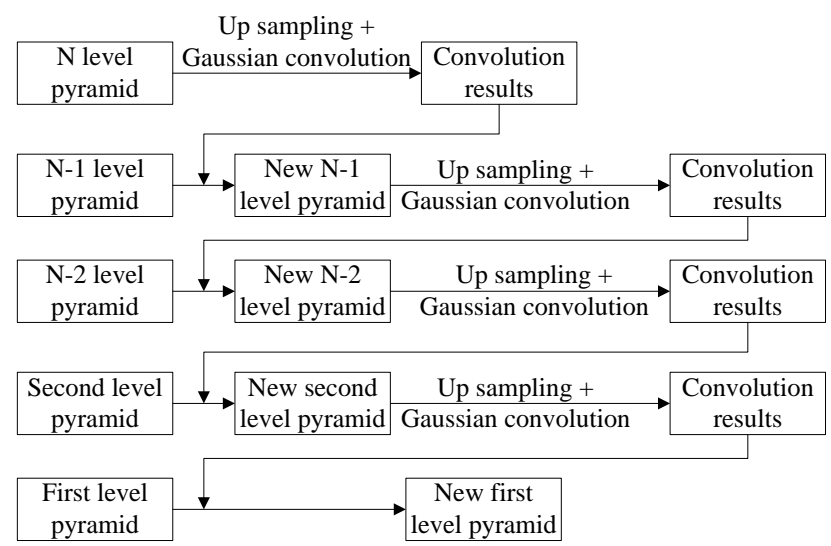

Fig. 5. Flowchart of brightness reconstruction based on the Laplacian pyramid

\section{Art Design Education}

\subsection{Current status of art design education and existing problems}

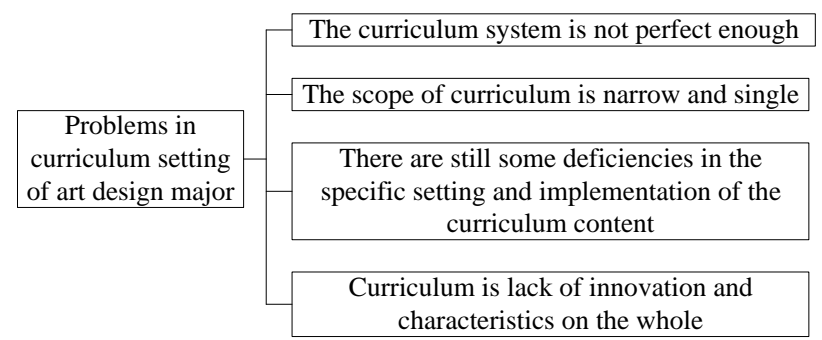

Fig. 6. Problems in the curriculum setting of the art design major

The art design education in the colleges and universities in China has experienced different development stages such as pattern design, arts and crafts, and art design, and through this process, the education model and the curriculum system in colleges and universities have undergone tremendous changes [32]. Art and design education is based on art and images, and its connection with economy and technology is often ignored [33]. At present, due to the negligence to the art design major in colleges and universities, the art design education in China remains standstill and marginalized, 
and art design is more treated like a mere mechanical skill. Figure 6 shows the existing problems in the curriculum setting of the art design major, including the incompleteness and the narrow scope of the curriculum, the deficiencies in the specific setting and implementation of the curriculum content, and the lack of features in the curriculum on the whole.

\subsection{Analysis on the innovation teaching in art design education}

\begin{tabular}{|c|}
$\begin{array}{c}\text { Physiological } \\
\text { needs }\end{array}$ \\
balance, secretion
\end{tabular}

Fig. 7. Maslow's hierarchy of needs

\begin{tabular}{|c|c|}
\hline $\begin{array}{c}\text { The social responsibility of art design professional } \\
\text { designer } \\
\text { professional } \\
\text { quality content }\end{array}$ & $\begin{array}{c}\text { Specialized designers of art design should have a more } \\
\text { in-depth and correct understanding of culture, and } \\
\text { adopt a correct attitude }\end{array}$ \\
\hline $\begin{array}{c}\text { The designer of art design profession should have } \\
\text { correct way of thinking, have the quality that grasps to } \\
\text { the thing comprehensively and whole }\end{array}$ \\
The professional designer of art design should have the \\
technical ability to externalize the design idea \\
comprehensively
\end{tabular}

Fig. 8. Professional requirements for a good art designer.

Figure 7 shows Maslow's hierarchy of needs. This hierarchy consists of five tiers, which, from the lowest to the highest, are physiological needs, safety, love and belonging, esteem and self-actualization. Most people put self-transcendence as part of the self-actualization needs. People's requirement for space is no longer part of their physiological needs, but has risen to a kind of emotional pursuit and identification they hope that the space can satisfy their current psychological feelings and be in line with their taste and identity. Figure 8 shows the professional requirements for a good 
art designer. First of all, a good designer should have a sense of social responsibility and put his or her humanistic care into the art design so that the art design will have positive effects on social development; secondly, the designer should have a profound and correct understanding of and take a proper attitude towards his or her national culture; thirdly, the designer should have the right way of thinking and be able to look at things from an overall perspective; and fourthly, the designer should have the technical ability to externalize the design ideas comprehensively. Figure 9 shows the principles for and stages of innovation teaching in art design education. The main principles include conformity, student-centeredness, and overall optimization; and innovation teaching is divided into basic, professional and subject-based innovation teaching. The innovation teaching of art design can be divided into three stages: cognitive stage, comprehension stage, and creative practice.

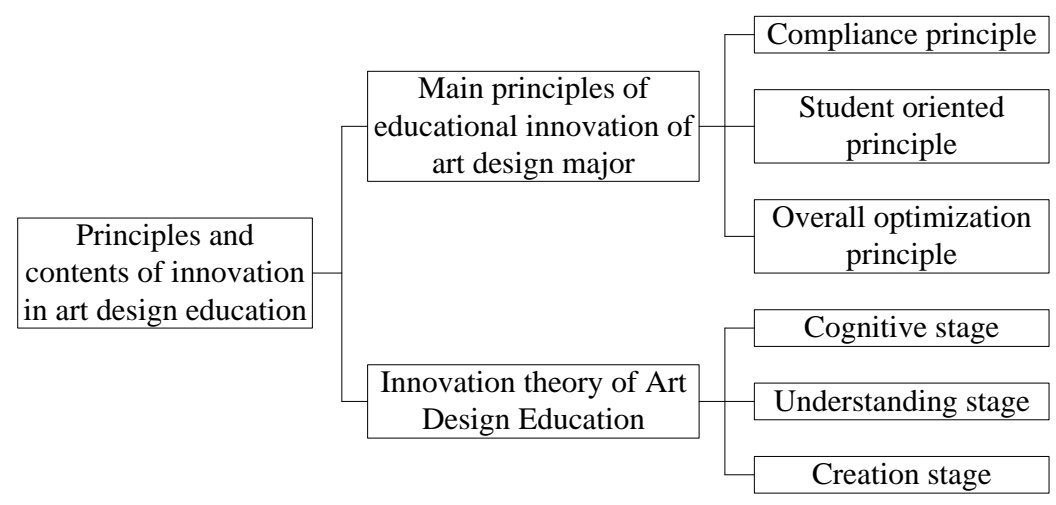

Fig. 9. Principles for and stages of innovation teaching in art design education

\section{Application of the Dynamic Image Fusion Technology in the Development of the Innovation Capabilities of College Students Majoring in Art Design}

\subsection{Development of the innovation capabilities of college students majoring in art design}

Art design is a comprehensive and also practical major. In addition to regular classroom teaching, art design education in colleges also pays more and more attention to the development of student's practical abilities. However, the environment for development of practical abilities is relatively complex, subject this kind of education to many factors. In conventional art design, the designer carries out planning and conceive design ideas, starting from the functional requirements, and finally implements the design and materialize the conception to satisfy consumers or customers. Figure 10 shows the operating mechanism and process of innovative talent development evaluation - first, determine the teaching objectives, perform teaching analysis and also learners and scenarios analysis, and write behavioral performance goals; then 
develop evaluation tools and teaching strategies, and develop and select teaching materials, and finally evaluate the teaching design and implementation, and give summative evaluation.

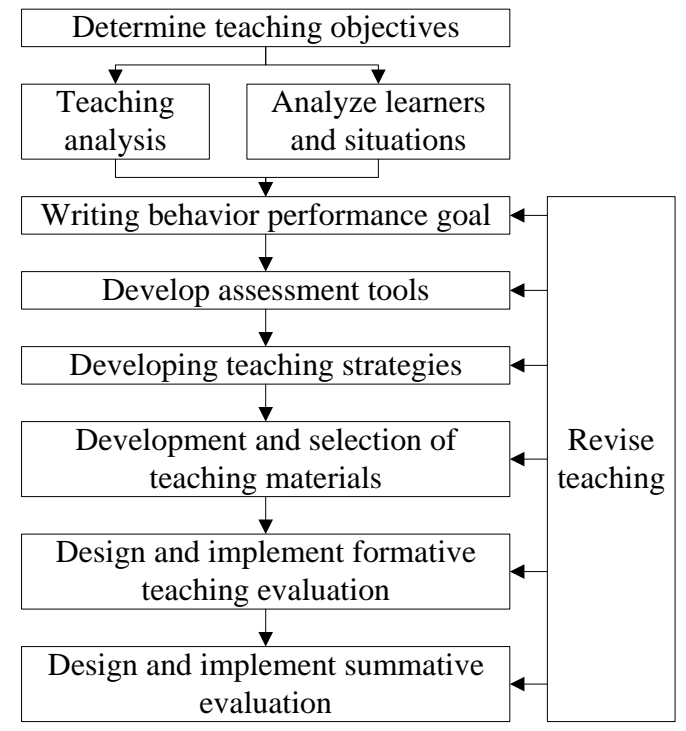

Fig. 10.Operating mechanism and process of innovative talent development evaluation

4.2 Analysis on the application of the dynamic image fusion technology in the development of the innovation capabilities
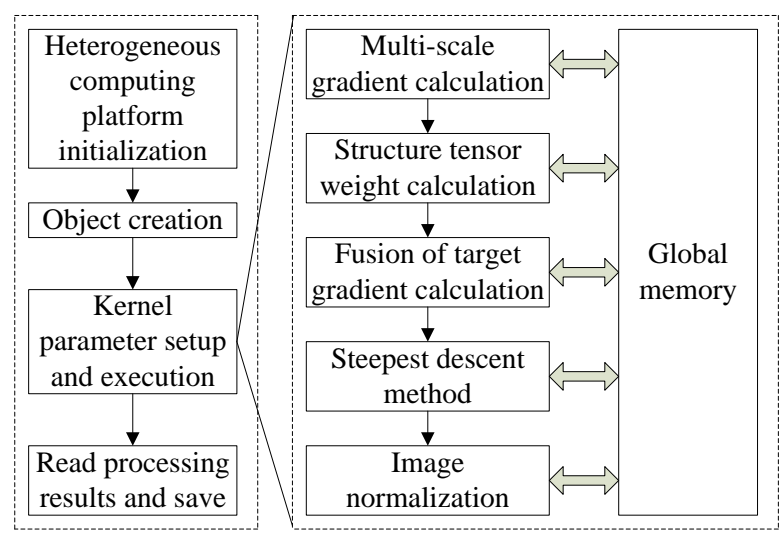

Fig. 11. Block diagram of how the image fusion algorithm is implemented on the field-programmable gate array platform 
Art design, as an important part of social life, is changing together with it in the information age, where knowledge is being updated increasingly faster every day. Figure 11 is a block diagram of how the image fusion algorithm is implemented on the field-programmable gate array platform, where the heterogeneous computing platform initialization, the object creation, the kernel parameter setting and execution and the processing result reading and saving modules are all host programs. Specifically, the heterogeneous computing platform initialization module initializes the CPU and the context required for the execution of the field-programmable gate array kernel; the created objects mainly include the program object used to store the kernel program and the kernel object used to call the kernel, the cache object used to cache images and other data buffer objects and the command transmission channels. The dynamic image fusion enhancement algorithm is mainly divided into five functional modules, which are used to calculate multi-scale gradient, structure tensor, and target gradient for the fused image, solve the steepest descent method and output normalized images, respectively.

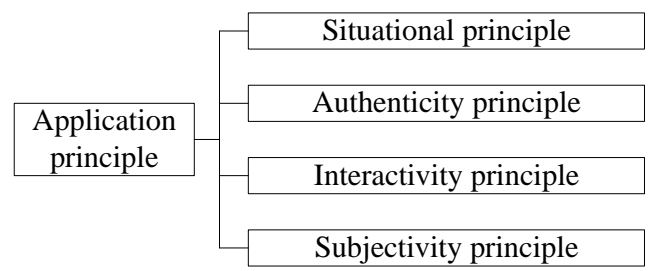

Fig. 12. Principles for application of the dynamic image fusion technology in the development of the innovation capabilities of college students majoring in art design

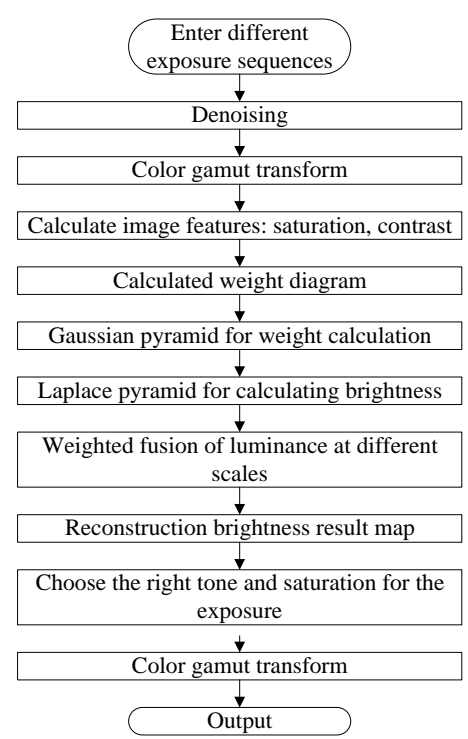

Fig. 13. Flow chart of the multi-exposure fusion algorithm for noisy images 
Figure 12 shows the principles for the application of the dynamic image fusion technology in the development of the innovation capabilities of college students majoring in art design, namely situationality, authenticity, interactivity, and subjectivity. Figure 13 is a flow chart of the multi-exposure fusion algorithm for noisy images, which consists of a number of steps, namely de-noising and transforming the color gamut of image sequences with different exposures, calculating the saturation and contrast of the images, the weight map, the Gaussian pyramid of the weights and the Laplacian Pyramid of brightness, fusing the images on a weighted basis at different scales according to the brightness, reconstructing the brightness result map, and selecting the right hue and saturation for the appropriate exposure. Figure 14 shows the methods for developing the innovation capabilities of college students majoring in art design, which include carrying out interactive teaching in both virtual and actual situations, establishing differentiated practical learning models, customizing and constructing virtual practical environment, and designing participatory learning in virtual practice.

\begin{tabular}{|c|c|}
\hline $\begin{array}{c}\text { The developing } \\
\text { method of cultivating } \\
\text { creative ability of art } \\
\text { design specialty }\end{array}$ & $\begin{array}{c}\text { Developing the interactive teaching mode of the } \\
\text { combination of virtual and real situations }\end{array}$ \\
\hline $\begin{array}{c}\text { Customization and construction of Virtual } \\
\text { innovation environment }\end{array}$ \\
\hline Participatory learning in design virtual \\
innovation
\end{tabular}

Fig. 14. Methods for developing the innovation capabilities of college students majoring in art design

\section{Conclusion}

This paper studied the application of the dynamic image fusion technology in the development of the innovation capabilities of college students majoring in art design. The specific conclusions are as follows:

1. The core of the multi-exposure fusion implementation framework is to assign different weights to the image sequences with different exposures based on image features, and then fuse the brightness image sequences into an image with complete scene information and extended dynamic range based on the weights.

2. The main principles for innovation teaching in art design education include conformity, student-centeredness, and overall optimization; and innovation teaching is divided into basic, professional and subject-based innovation teaching. The innovation teaching of art design can be divided into three stages: cognitive stage, comprehension stage, and creative practice.

3. The heterogeneous computing platform initialization module initializes the CPU and the context required for the execution of the field-programmable gate array kernel; the created objects mainly include the program object used to store the ker- 
nel program and the kernel object used to call the kernel, the cache object used to cache images and other data buffer objects and the command transmission channels.

\section{References}

[1] Shah, S.A.A., Habib, N., Nadeem, M.S.A., Alshdadi, A.A., Alqarni, M., Aziz, W. (2020). Extraction of dynamical information and classification of heart rate variability signals using scale-based permutation entropy measures. Traitement du Signal, 37(3): 355-365. https://doi.org/10.18280/ts.370302

[2] Fekri-Ershad, S. (2019). Gender classification in human face images for smart phone applications based on local texture information and evaluated Kullback-Leibler divergence. Traitement du Signal, 36(6): 507-514. https://doi.org/10.18280/ts.360605

[3] Guo, R., Shen, X., Zhang, X. (2020). Comprehensive measure for evaluating image fusion algorithm. Journal of Electronic Imaging, 29(1): 013008. https://doi.org/10.1117/1.JEI.29. $\underline{1.013008}$

[4] Heidari, S., Naseri, M., Gheibi, R., Baghfalaki, M., Pourarian, M. R., Farouk, A. (2017). A new quantum watermarking based on quantum wavelet transforms. Communications in theoretical Physics,67(6), 732. https://doi.org/10.1088/0253-6102/67/6/732

[5] Batle, J., Ooi, C. R., Farouk, A., Abdalla, S. (2016). Nonlocality in pure and mixed n-qubit X states. Quantum Information Processing,15(4), 1553-1567. https://doi.org/10.1007/s11 $\underline{128-015-1216-5}$

[6] Xiao, X.H., Xie, J.G., Niu, J.P., Cao, W. (2020). A novel image fusion method for water body extraction based on optimal band combination. Traitement du Signal, 37(2): 195-207. https://doi.org/10.18280/ts.370205

[7] Zhou, H., Peng, J., Liao, C., Li, J. (2020). Application of deep learning model based on image definition in real-time digital image fusion. Journal of Real-Time Image Processing, 17: 1-12. https://doi.org/10.1007/s11554-020-00956-1

[8] Ma, Z., Guan, J., Li, R.Y.M. (2020). Research on Innovative Teaching Mode of Art Education in the Age of Convergence of Media, International Journal of Emerging Technologies in Learning, 16(2): 272-284.

[9] Liu, Q., Chen, H., C. Crabbe, M.J. (2020). Interactive Study of Multimedia and Virtual Technology in Art Education, International Journal of Emerging Technologies in Learning, 16(1): 80-93. https://doi.org/10.3991/ijet.v16i01.18227

[10] Gao, Z., Yang, M., Xie, C. (2017). Space target image fusion method based on image clarity criterion. Optical engineering, 56(5): 053102. https://doi.org/10.1117/1.OE.56.5.05 $\underline{3102}$

[11] Zhao, W., Ma, X., Liang, L., Liang, L., Yang, Y., Yang, K., Ong, S.H. (2019). Remote sensing image registration based on dynamic threshold calculation strategy and multiplefeature distance fusion. IEEE Journal of Selected Topics in Applied Earth Observations and Remote Sensing, 12(10): 4049-4061. https://doi.org/10.1109/JSTARS.2019.2938622

[12] Li, J., Li, Y. (2019). Research and application of computer aided design system for product innovation. Journal of Computational Methods in Sciences and Engineering, 19(S1): 4146. https://doi.org/10.3233/JCM-191006

[13] Lee, K.Y., Ku, N., Cha, J.H. (2013). Undergraduate courses for enhancing design ability in naval architecture. International Journal of Naval Architecture and Ocean Engineering, 5(3): 364-375. https://doi.org/10.2478/IJNAOE-2013-0139 
[14] Gholami, A., Wahid, M.A., Mohammed, H.A. (2017). Thermal-hydraulic performance of fin-and-oval tube compact heat exchangers with innovative design of corrugated fin patterns. International Journal of Heat and Mass Transfer, 106: 573-592. https://doi.org/10. 1016/j.ijheatmasstransfer.2016.09.028

[15] Som, A. (2012). Organizational response through innovative HRM and re-design: a comparative study from France and India. The International Journal of Human Resource Management, 23(5): 952-976. https://doi.org/10.1080/09585192.2012.651323

[16] Fu, Y.Z., Zhu, Y.F. (2016). Curriculum system construction for art and design major in the transition between secondary and higher vocational education. Journal of Landscape Research, 8(6): 124-126. https://doi.org/10.16785/jissn1943-989x.2016.6.029

[17] Zhao, L., Song, J., Babu, P., Palomar, D.P. (2016). A unified framework for low autocorrelation sequence design via majorization-minimization. IEEE Transactions on Signal Processing, 65(2): 438-453. https://doi.org/10.1109/TSP.2016.2620113

[18] Arora, A., Tsinos, C.G., Rao, B.S.M.R., Chatzinotas, S., Ottersten, B. (2019). Hybrid transceivers design for large-scale antenna arrays using majorization-minimization algorithms. IEEE Transactions on Signal Processing, 68: 701-714. https://doi.org/10.1109 /TSP.2019.2957611

[19] Chen, G., Li, L., Jin, W., Li, S. (2019). High-dynamic range, night vision, image-fusion algorithm based on a decomposition convolution neural network. IEEE Access, 7: 169762169772. https://doi.org/10.1109/ACCESS.2019.2954912

[20] Guo, Q., Zhao, C., Escano, L.I., Young, Z., Xiong, L., Fezzaa, K., Chen, L. (2018). Transient dynamics of powder spattering in laser powder bed fusion additive manufacturing process revealed by in-situ high-speed high-energy x-ray imaging. Acta Materialia, 151: 169-180. https://doi.org/10.1016/j.actamat.2018.03.036

[21] Dev, S., Savoy, F.M., Lee, Y.H., Winkler, S. (2018). High-dynamic-range imaging for cloud segmentation. Atmospheric Measurement Techniques, 11(4): 2041-2049. https://doi. org/10.5194/amt-11-2041-2018

[22] Liu, Y., Zhou, D., Nie, R., Hou, R., Ding, Z. (2020). Construction of high dynamic range image based on gradient information transformation. IET Image Processing, 14(7): 13271338. https://doi.org/10.1049/iet-ipr.2019.0118

[23] Fan, Y., Han, H., Tang, Y., Zhi, T. (2019). Dynamic objects elimination in SLAM based on image fusion. Pattern Recognition Letters, 127: 191-201. https://doi.org/10.1016/j.patr ec.2018.10.024

[24] Ramakrishnan, V., Pete, D.J. (2021). Savitzky-Golay Filtering-Based Fusion of Multiple Exposure Images for High Dynamic Range Imaging. SN Computer Science, 2(3): 1-10. https://doi.org/10.1007/s42979-021-00594-9

[25] Li, B., Peng, H., Wang, J., Huang, X. (2020). Multi-focus image fusion based on dynamic threshold neural $\mathrm{P}$ systems and surfacelet transform. Knowledge-Based Systems, 196: 105794. https://doi.org/10.1016/j.knosys.2020.105794

[26] Gao, H., Chen, Q., Liu, C., Gu, G. (2021). High dynamic range infrared image acquisition based on an improved multi-exposure fusion algorithm. Infrared Physics \& Technology, 115: 103698. https://doi.org/10.1016/j.infrared.2021.103698

[27] Calta, N.P., Thampy, V., Lee, D.R., Martin, A.A., Ganeriwala, R., Wang, J., Matthews, M.J. (2020). Cooling dynamics of two titanium alloys during laser powder bed fusion probed with in situ X-ray imaging and diffraction. Materials \& Design, 195: 108987. https://doi.org/10.1016/j.matdes.2020.108987

[28] Kim, B.H., Bohak, C., Kwon, K.H., Kim, M.Y. (2020). Cross fusion-based low dynamic and saturated image enhancement for infrared search and tracking systems. IEEE Access, 8: 15347-15359. https://doi.org/10.1109/ACCESS.2020.2966794 
[29] Setumin, S., Aminudin, M.F.C., Suandi, S.A. (2020). Canonical Correlation Analysis Feature Fusion with Patch of Interest: A Dynamic Local Feature Matching for Face Sketch Image Retrieval. IEEE Access, 8: 137342-137355. https://doi.org/10.1109/ACCESS.2020. $\underline{3009744}$

[30] Gholami, S., Jaferzadeh, K., Shin, S., Moon, I. (2019). An efficient image-based verification scheme by fusion of double random phase encoding and dynamic chaotic map. Multimedia Tools and Applications, 78(17): 25001-25018. https://doi.org/10.1007/s11042019-7714-1

[31] Sun, T. (2020). Probing Ultrafast Dynamics in Laser Powder Bed Fusion Using HighSpeed X-Ray Imaging: A Review of Research at the Advanced Photon Source. Jom, 72(3): 999-1008. https://doi.org/10.1007/s11837-020-04015-9

[32] Krishnamoorthy, S., Punithavathani, S., Priya, J.K. (2017). Extraction of well-exposed pixels for image fusion with a sub-banding technique for high dynamic range images. International Journal of Image and Data Fusion, 8(1): 54-72. https://doi.org/10.1080/194 79832.2016.1226967

[33] Du, L., Sun, H.Y., Zhang, T.H., Wang, S. (2015). High dynamic range image fusion based on camera response function. Computer Engineering \& Science, 37(7): 1331-1337. https ://doi.org/10.3969/j.issn.1007-130X.2015.07.017

\section{$7 \quad$ Author}

Zhe Wang is a lecturer in School of Arts, Jiaozuo University, Han nationality, majoring in art design theory, member of Jiaozuo Art Design Association. wz15839 128961@163.com

Article submitted 2021-04-13. Resubmitted 2021-05-28. Final acceptance 2021-05-30. Final version published as submitted by the authors. 